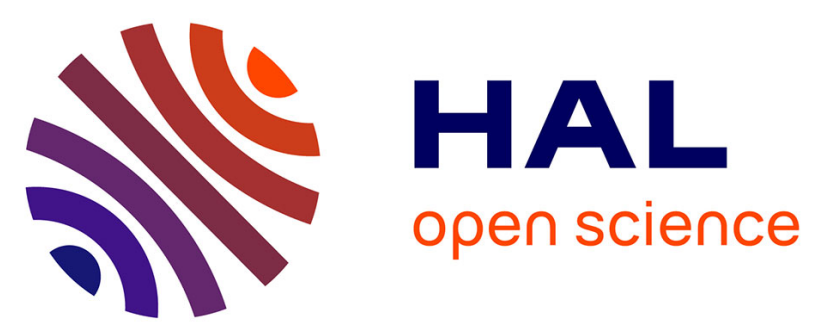

\title{
Analysis of the whispering gallery mode sapphire Fe3+ maser under magnetic field
}

M. Mrad, P.-Y. Bourgeois, M. E. Tobar, Y. Kersalé, V. Giordano

\section{To cite this version:}

M. Mrad, P.-Y. Bourgeois, M. E. Tobar, Y. Kersalé, V. Giordano. Analysis of the whispering gallery mode sapphire Fe3+ maser under magnetic field. European Physical Journal: Applied Physics, 2012, 57 (2), pp.21005. 10.1051/epjap/2012110366 . hal-00686948

\section{HAL Id: hal-00686948 https://hal.science/hal-00686948}

Submitted on 5 May 2021

HAL is a multi-disciplinary open access archive for the deposit and dissemination of scientific research documents, whether they are published or not. The documents may come from teaching and research institutions in France or abroad, or from public or private research centers.
L'archive ouverte pluridisciplinaire HAL, est destinée au dépôt et à la diffusion de documents scientifiques de niveau recherche, publiés ou non, émanant des établissements d'enseignement et de recherche français ou étrangers, des laboratoires publics ou privés. 


\title{
Analysis of the whispering gallery mode sapphire $\mathrm{Fe}^{3+}$ maser under magnetic field
}

\author{
M. Mrad ${ }^{1}$, P.Y. Bourgeois ${ }^{1}$, M.E. Tobar ${ }^{2}$, Y. Kersalé $^{1, a}$, and V. Giordano ${ }^{1}$ \\ ${ }^{1}$ FEMTO-ST Institute, Time and Frequency Department, UMR 6174 CNRS, ENSMM, 26 Chemin de l'Épitaphe, \\ 25030 Besançon Cedex, France \\ ${ }^{2}$ University of Western Australia, School of Physics, Crawley 6009, WA, Australia
}

\begin{abstract}
This article describes the influence of the magnetic field on a small amount of residual $\mathrm{Fe}^{3+}$ ion impurities contained in a sapphire resonator excited on a whispering gallery mode and cooled down to $4 \mathrm{~K}$. The energy levels and transition probabilities between pairs of levels of the system are calculated in order to determine the effect of the magnetic field on the maser output power and also on the pump power threshold.
\end{abstract}

\section{Introduction}

The need for microwave sources exhibiting ultra-high spectral purity and high temporal stability is a key point highlighted by the performance limitations in major strategic applications such as radar, spatial communications, ultrahigh accuracy navigation system and high bitrate optical telecommunications. The Cryogenic Sapphire Oscillator (CSO) is currently the only microwave technology able to provide a relative frequency stability better than $1 \times 10^{-14}$ for integration time between $1 \mathrm{~s}$ and $10^{4} \mathrm{~s}[1,2]$. Nevertheless, the CSO remains a complex instrument and needs several servo loops to control the oscillator phase and the microwave power incident on the resonator. Recently, we reported in $[3,4]$ the possibility to use the sapphire resonator in another way. Here, the sustaining amplification is achieved through the interaction between a whispering gallery mode and the paramagnetic $\mathrm{Fe}^{3+}$ ions also present in the sapphire crystal. The frequency instability of the Whispering Gallery Mode maser is limited to $10^{-14}$ due to the measurement readout [5].

In the sapphire crystal, the $\mathrm{Fe}^{3+}$ ion exhibits three energy states at zero magnetic field enabling to realize a self-sustaining solid-state maser. For a maser the ultimate limit in terms of frequency instability is the SchawlowTownes limit [6] which is inversely proportional to the square root of the maser output power. As a consequence to decrease the frequency instability we have to increase the maser output power.

In this paper we describe, theoretically, the effect of a magnetic field on the Whispering Gallery Mode (WGM) maser oscillator and we show that in a particular

\footnotetext{
a e-mail: yann.kersale@femto-st.fr
}

configuration the maser output power can be increased by a factor of $6 \mathrm{~dB}$.

\section{Principle}

Maser materials, and maser operations were introduced in the 1950s by several groups [7-10]. In particular, the action of iron ions in corundum was studied by Friedman and Nagy [11]. The corundum used for this experiment was of poor quality, and as a consequence the iron doping was deliberately strong. Recently we have demonstrated that high-quality sapphire resonator with a small amount of paramagnetic impurities $\left(\mathrm{Fe}^{3+}\right.$ ions) can provide a maser signal with an output power of $-30 \mathrm{dBm}$ [12]. The maser fundamental limit is defined by $[6,13]$ :

$$
\sigma_{y}(\tau)=\frac{1}{Q_{\text {signal }}} \sqrt{\frac{k_{B} T}{2 P_{\text {out }} \tau}}
$$

with $Q_{\text {signal }}$ being the loaded $Q$-factor of the considered mode, $P_{\text {out }}$ the power in watts at the maser output, $k_{B}$ the Boltzmann constant and $T$ the temperature of the amplifying medium. As we can see in equation (1) to get the best frequency instability we have to maximize the maser output power. In order to maximize it we have studied the effect of the magnetic field (orientation and amplitude) on the pump power threshold and on the maser output power.

\section{$2.1 \mathrm{Fe}^{3+}$ Characteristics}

The $\mathrm{Fe}^{3+}$ ion has the $[\mathrm{Ar}] 3 d^{5}$ electronic configuration. The number of total spins is $S=5 / 2$, the total orbital number 
is $L=0$ and the spectroscopic ground state term is ${ }^{6} S$. The $\mathrm{Fe}^{3+}$ ion replaces the cation $\mathrm{Al}^{3+}$ in the crystal and undergoes the action of a crystal field of the nearby ions $\mathrm{O}^{2-}$ and $\mathrm{Al}^{3+}$.

These interactions are described by the following Hamiltonian:

$$
\begin{aligned}
\widehat{H}= & g \mu_{B} H \hat{S}+D\left[\hat{S}_{Z}^{2}-\frac{1}{3} S(S+1)\right] \\
& +\frac{1}{6} a\left[\hat{S}_{\xi}^{4}+\hat{S}_{\eta}^{4}+\hat{S}_{\zeta}^{4}-\frac{1}{5} S(S+1)\left(3 S^{2}+3 S-1\right)\right] \\
& +\frac{1}{180} F\left[35 \hat{S}_{Z}^{4}-30 S(S+1) \hat{S}_{Z}^{2}\right. \\
& \left.+25 \hat{S}_{Z}^{2}-6 S(S+1)+3 S^{2}(S+1)\right] .
\end{aligned}
$$

To calculate this Hamiltonian we have considered the crystal cubic field with trigonal distortion and the axis $(Z)$ as axis of symmetry, which is the case of our resonators. Indeed, corundum $\alpha-\mathrm{Al}_{2} \mathrm{O}_{3}$ crystallizes in the rhombohedral system. $(\xi, \eta, \zeta)$ are the axes of the trigonal crystal field. The crystalline axis of symmetry $\left(C_{3}\right)=(Z)$ is defined in the direction $(1,1,1)$ of this system. For more details on the structure of corundum, see $[8,9]$.

The energy levels are calculated based on the parameters " $D$ ", " $F$ " and " $a$ " in the above equation. " $D$ " and " $F "$ are the parameters of the axial field, respectively, second and fourth order, and the spacing between levels is primarily due to " $D$ ". " $a$ " is the parameter of trigonal field (mixing between the levels $E_{1}$ and $E_{3}$ of the system). The values of these parameters, measured at $4 \mathrm{~K}$ by Symmons and Bogle [14], are equal to: $D=1719.2 \pm 1,|a|=229.4 \pm 1$ and $a-F=341.5 \pm 1$ expressed in $10^{-4} \mathrm{~cm}^{-1}$. We assumed that $h_{i}=g_{j} \times \mu_{B} \times H_{i}$, where the indices are respectively $i=x, y$ and $Z$ and $j=\perp, \perp$ and $\|, g$ is the Lande factor and $\mu_{B}$ the Bohr magneton. We will also consider that $h^{ \pm}=h_{x} \pm i h_{y}=g_{\perp} \times \mu_{B}\left(H_{x} \pm i H_{y}\right), \gamma=a-F$ and $p=(2 \sqrt{5} / 3) a$. Moreover for our system $(S=5 / 2)$ the factor $\frac{1}{3} S(S+1)=35 / 12$.

The matrix Hamiltonian of the $\mathrm{Fe}^{3+}$ ion in sapphire can now be written as follows: see equation (3) in the next page.

\subsection{Energy levels}

The matrix in equation (3) was numerically solved in order to determine the eigenstates and eigenvalues of this system. In order to verify if our numerical model is correct we plot (Fig. 1) the evolution of the energy levels as a function of a magnetic field applied in the parallel direction of the crystal axis.

The energy levels of the $\mathrm{Fe}^{3+}$ ions in the sapphire lattice represented here are very similar to what has been published before [15] and so we can consider that our numerical model is correct.

In the next plot we have represented the energy levels for a magnetic field perpendicular to the crystal axis (see Fig. 2).



Fig. 1. (Color online) Energy level variations of the $\mathrm{Fe}^{3+}$ ion in alumina as a function of a magnetic field parallel to the crystal Z-axis.

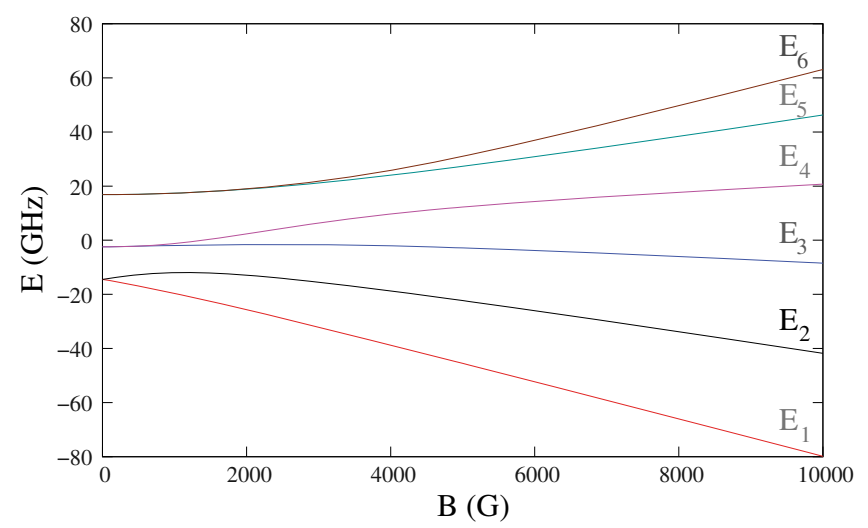

Fig. 2. (Color online) Energy levels variations of the $\mathrm{Fe}^{3+}$ ion in alumina as a function of a magnetic field perpendicular to the crystal $Z$-axis.

When the magnetic field is parallel to the $Z$-axis all the levels are immediately split when the value of the field increases. As opposed, when the field is perpendicular to the crystal axis, and with a value of $50 \mathrm{G}$, states 5 and 6 are separated by $450 \mathrm{kHz}$ as well as levels 3 and 4 while states 1 and 2 are separated by more than $400 \mathrm{MHz}$. Considering the Electronic Spin Resonance (ESR) bandwidth of $100 \mathrm{MHz}$ [12] we can consider that the two upper states (i.e., levels 5 and 6 ) are mixed and the two lower states can be considered completely separated and so on, ions pumped from the level 1 to levels 5 and 6 will participate in the maser action.

A zoom on the prevision plot leads us to determine the transition frequencies of the system under magnetic field (see Fig. 3).

The transition frequencies of the system with a magnetic field of $50 \mathrm{G}$ oriented in the perpendicular plane of the crystal are a little bit different from the values with zero magnetic field. We get: $\nu_{14}=12.2676 \mathrm{GHz}$ $\left(12.037 \mathrm{GHz}\right.$ with no magnetic field) and $\nu_{16}=$ $31.5647 \mathrm{GHz}$ (31.318 GHz with no magnetic field). We will now assume that our sapphire resonator have whispering gallery modes centered on the $\nu_{14}$ transition and another one centered between $\nu_{15}$ and $\nu_{16}$ at $31.5645 \mathrm{GHz}$. 


$$
\hat{H}=\left|\begin{array}{cccccc}
-\frac{5}{2} h_{Z}-\frac{1}{3} \gamma+\frac{10}{3} D & \frac{\sqrt{5}}{2} h^{+} & 0 & \pm i p & 0 & 0 \\
\frac{\sqrt{5}}{2} h^{-} & -\frac{3}{2} h_{Z}+\gamma-\frac{2}{3} D & \sqrt{2} h^{+} & 0 & 0 & 0 \\
0 & \sqrt{2} h^{-} & -\frac{1}{2} h_{Z}-\frac{2}{3} \gamma-\frac{8}{3} D & \frac{3}{2} h^{+} & 0 & \mp i p \\
\mp i p & 0 & \frac{3}{2} h^{-} & \frac{1}{2} h_{Z}-\frac{2}{3} \gamma-\frac{8}{3} D & \sqrt{2} h^{+} & 0 \\
0 & 0 & 0 & \sqrt{2} h^{-} & \frac{3}{2} h_{Z}+\gamma-\frac{2}{3} D & \frac{\sqrt{5}}{2} h^{+} \\
0 & 0 & \pm i p & 0 & \frac{\sqrt{5}}{2} h^{-} & \frac{5}{2} h_{Z}-\frac{1}{3} \gamma+\frac{10}{3} D
\end{array}\right|
$$

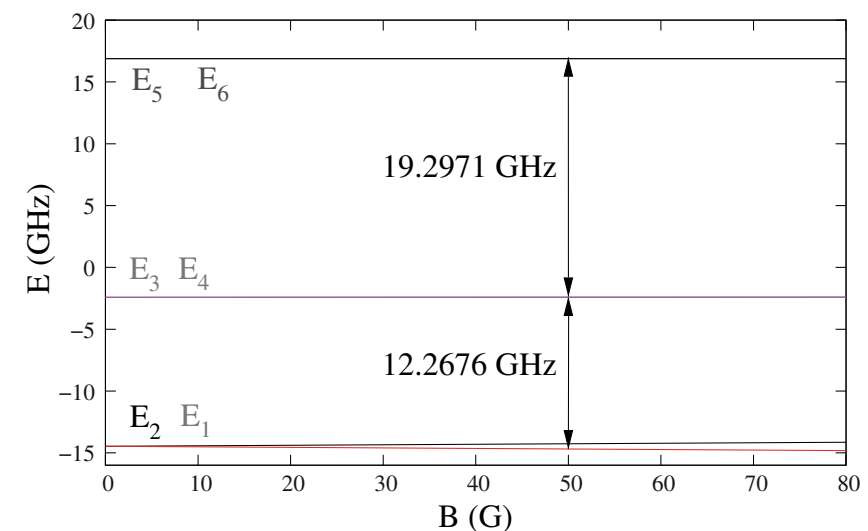

Fig. 3. (Color online) Zoom on the energy levels variations of the $\mathrm{Fe}^{3+}$ ion in alumina as a function of a magnetic field perpendicular to the crystal $Z$-axis.

These can be realized by machining the radius of our actual resonator centered at $12.037 \mathrm{GHz}$ and $31.318 \mathrm{GHz}$. Under this configuration we consider now that the maser output signal will operate between levels 4 and 1 and the pump signal will be applied between the level 1 and the mixed states 5 and 6 .

\section{General populations of the system under field}

\subsection{Population differences at thermal equilibrium}

When there is no magnetic field applied, the $\mathrm{Fe}^{3+}$ ion gets three energy levels in the sapphire lattice and with an applied magnetic field each state splits in two states with respect to the Zeeman effect. At thermal equilibrium the level populations have the Boltzmann relationships [10]:

$$
\frac{N_{j}}{N_{i}}=\exp \left(-\frac{h \nu_{i j}}{k_{B} T}\right)=e^{-\Delta_{i j}} \text { and } \quad \sum_{i=1}^{6} N_{i}=N,
$$

where $N$ is the total number of spins and $\Delta_{i j}=-\Delta_{j i}$ with $i, j=1,2,3,4,5$ and 6 .

The population differences at the thermal equilibrium can thus be expressed as:

$$
\Delta N_{i j}=N_{i}-N_{j}=\frac{1-e^{-\Delta_{i j}}}{\sum_{k=1}^{6} e^{-\Delta_{i k}}} \times N .
$$

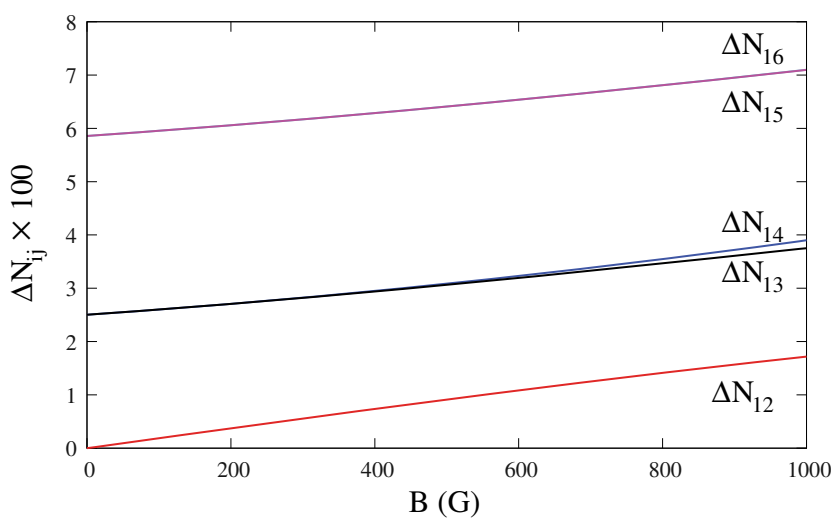

Fig. 4. (Color online) Population differences of the $\mathrm{Fe}^{3+}$ ion in alumina as a function of a magnetic field perpendicular to the crystal $Z$-axis.

Figure 4 represents the population differences at $4.2 \mathrm{~K}$ as a function of the magnetic field perpendicular to the crystal axis.

From this plot we get: $\Delta N_{12}=0.09332 \times 10^{-2} \times N$; $\Delta N_{13}=2.55064 \times 10^{-2} \times N ; \Delta N_{14}=2.55066 \times 10^{-2} \times N$; $\Delta N_{15}=5.90472 \times 10^{-2} \times N ; \Delta N_{16}=5.90479 \times 10^{-2} \times N$.

\subsection{Rate equation for a six-level system}

The rate equations of this system are expressed as follows:

$$
\begin{aligned}
& \frac{\mathrm{d} n_{1}}{\mathrm{~d} t}=-\sum_{j \neq 1}^{6} \frac{\Delta n_{1 j}-\Delta N_{1 j}}{2 \tau_{1}^{(1 j)}}-W_{S} \Delta n_{14}-W_{15} \Delta n_{15} \\
& -W_{16} \Delta n_{16} \text {, } \\
& \frac{\mathrm{d} n_{2}}{\mathrm{~d} t}=-\sum_{j \neq 2}^{6} \frac{\Delta n_{2 j}-\Delta N_{2 j}}{2 \tau_{1}^{(2 j)}}-W_{25} \Delta n_{25}-W_{26} \Delta n_{26}, \\
& \frac{\mathrm{d} n_{3}}{\mathrm{~d} t}=-\sum_{j \neq 3}^{6} \frac{\Delta n_{3 j}-\Delta N_{3 j}}{2 \tau_{1}^{(3 j)}} \\
& \frac{\mathrm{d} n_{4}}{\mathrm{~d} t}=-\sum_{j \neq 4}^{6} \frac{\Delta n_{4 j}-\Delta N_{4 j}}{2 \tau_{1}^{(4 j)}}+W_{S} \Delta n_{14}, \\
& \frac{\mathrm{d} n_{5}}{\mathrm{~d} t}=-\sum_{j \neq 5}^{6} \frac{\Delta n_{5 j}-\Delta N_{5 j}}{2 \tau_{1}^{(5 j)}}+W_{15} \Delta n_{15}+W_{25} \Delta n_{25}, \\
& \frac{\mathrm{d} n_{6}}{\mathrm{~d} t}=-\sum_{j \neq 6}^{6} \frac{\Delta n_{6 j}-\Delta N_{6 j}}{2 \tau_{1}^{(6 j)}}+W_{16} \Delta n_{16}+W_{26} \Delta n_{26},
\end{aligned}
$$


where $W_{i j}$ represents the rate absorption, and $\tau_{1}^{i j}$ the spin lattice relaxation time of the $\mathrm{Fe}^{3+}$ ion in the sapphire lattice between levels $i$ and $j$. We will then assume that $\tau_{1}^{i j}=\tau_{1} \forall i, j$ and is equal to $10 \mathrm{~ms}[8,16,17]$ at the liquid helium temperature.

To determine the steady state of this system $\left(\mathrm{d} n_{i} / \mathrm{d} t=\right.$ 0 ) we have to consider equation (7) which is the sum of population differences:

$$
\Delta n_{12}+\Delta n_{23}+\Delta n_{34}+\Delta n_{45}+\Delta n_{56}-\Delta n_{16}=0 .
$$

The maser output frequency and the stimulated emission rate (i.e., between levels 1 and 4 ) will be denoted by $\nu_{S}$ and $W_{S}$ and for the pump signal (i.e., from level 1 to levels 5 and 6 ) will be denoted by $\nu_{p}$ and $W_{p}$ with:

$$
\nu_{p}=\frac{\nu_{15}+\nu_{16}}{2} ; \quad W_{p}=\frac{W_{15}+W_{16}}{2} .
$$

Finally, the stationary solutions of the system are:

$$
\begin{aligned}
& \Delta n_{14}=\frac{-3\left(\Delta N_{15}+\Delta N_{16}\right) \tau_{1} W_{p}+9 \Delta N_{14}\left(1+\tau_{1} W_{p}\right)}{3\left(3+\left(3 W_{p}+2 W_{S}\right) \tau_{1}\right)+4 \tau_{1}^{2} W_{p} W_{S}}, \\
& \frac{\Delta n_{15}+\Delta n_{16}}{2} \\
& =\frac{9\left(\Delta N_{15}+\Delta N_{16}\right)+6\left(\Delta N_{15}+\Delta N_{16}-\Delta N_{14}\right) \tau_{1} W_{S}}{6\left(3+\left(3 W_{p}+2 W_{S}\right) \tau_{1}\right)+8 \tau_{1}^{2} W_{p} W_{S}} .
\end{aligned}
$$

\section{The maser pump and output power}

In this section we will define the relation between the pump power $\left(P_{p}\right)$ and the maser output power $\left(P_{41}\right)$.

\subsection{Evolution of the emitted power as a function of pump power}

The maximum of power that can be emitted by a maser system is defined as [10]

$$
P_{41}=h \nu_{S} W_{S} \Delta n_{41} V_{41},
$$

where $V_{41}$ is the effective volume of the whispering gallery mode involved in the emission process [18].

The stimulated absorption is represented as follows [10]:

$$
W_{S}=Q_{0_{S}} \frac{1}{4} \gamma^{2} g\left(\nu_{14}\right) H_{\text {acsignal }}^{2} \sigma_{14}^{2}
$$

From the definition of the Poynting vector, the power in the resonator is directly proportional to the magnetic field. It leads to:

$$
H_{\text {acsignal }}^{2}=\frac{2 P_{41}}{Z_{c} S_{e f f_{14}}} .
$$

Therefore:

$$
W_{S}=Q_{0_{S}} \frac{\gamma^{2} g\left(\nu_{14}\right) \sigma_{14}^{2}}{2 Z_{c} S_{e f f_{14}}} P_{41}=Q_{0_{S}} w_{S_{0}} P_{41} .
$$

This representation can also be applied to the probability of absorption:

$$
W_{p}=Q_{0_{p}} \frac{\gamma^{2} Q_{0_{p}} g\left(\nu_{p}\right) \sigma_{p}^{2}}{2 Z_{c} S_{e f f_{p}}} P_{p}=Q_{0_{p}} w_{p_{0}} P_{p} .
$$

From equation (7) we can write:

$$
w_{p_{0}}=\frac{w_{15_{0}}+w_{16_{0}}}{2}=\frac{1}{2} \frac{\gamma^{2} Q_{0_{p}} g\left(\nu_{p}\right)\left(\sigma_{15}^{2}+\sigma_{16}^{2}\right)}{2 Z_{c} S_{e f f_{p}}},
$$

where $\gamma$ is the gyromagnetic factor. $S_{e f f_{14}}$ and $S_{\text {eff }}$ are respectively the effective surfaces of the whispering gallery mode in the meridian resonator plane for the maser signal and the maser pump, $Z_{c}$ is the characteristic resonator impedance $(112 \Omega)$, and $Q_{0_{S}}$ and $Q_{0_{p}}$ are the quality factors of the whispering gallery modes for the maser signal and the maser pump respectively [18].

From equation (9) we can now determine the maser output power $\left(P_{41}\right)$ as a function of the pump power $\left(P_{p}\right)$, with $C=h \nu_{14} w_{S_{0}} \Delta N_{41} V_{14}$ : see equation (17) in the next page.

\subsection{Pump power threshold}

The pump power threshold $P_{p_{t h}}$ appears when the population inversion occurs $\left(\Delta n_{14}=0\right)$. The rate absorption threshold $\left(W_{p_{T h}}\right)$ is the solution of the following equation (from Eq. (8)):

$$
3 \tau_{1} W_{p_{T h}}\left(3 \Delta N_{14}-\left(\Delta N_{15}+\Delta N_{16}\right)\right)+9 \Delta N_{14}=0 .
$$

Finally we get:

$$
W_{p_{T h}}=\frac{3}{\tau_{1}} \frac{\Delta N_{14}}{-3 \Delta N_{14}+\Delta N_{15}+\Delta N_{16}}=w_{p_{0}} P_{p_{T h}} .
$$

\subsection{Determination of the transition probabilities and the line shape function}

In order to determine the transition probabilities $\left(W_{S}\right.$ and $W_{p}$ ) of the maser and pump transitions, we have to solve the previous Hamiltonian in order to calculate $\sigma_{i j}^{2}$ which characterizes the strength of the transition probabilities. On the next plots we have represented the evolution of all the transition probabilities for a magnetic field perpendicular to the crystal axis (see Figs. 5-8).

In reality, if the signal is not exactly at the transition frequency, the transition probability decreases as determined by the line shape function $g(\nu)$. The line shape function is usually described by a Lorentzian or a Gaussian profile. It is a function normalized and centered around the transition frequency:

$$
\int_{0}^{\infty} g(\nu) \mathrm{d} \nu=1
$$

In our case, we have proved that the $\mathrm{Fe}^{3+}$ ions are inhomogeneously broadened [12] and so $g(\nu)$ is represented by 


$$
P_{41}=\frac{3\left(-3\left(1+P_{p} \tau_{1} Q_{0_{p}} w_{p_{0}}\right)+C\left(\left(\Delta N_{15}+\Delta N_{16}\right) P_{p} \tau_{1} Q_{0_{p}} w_{p_{0}}-3 \Delta N_{14}\left(1+P_{p} \tau_{1} Q_{0_{p}} w_{p_{0}}\right)\right)\right)}{2 \tau_{1}\left(3+2 P_{p} \tau_{1} Q_{0_{p}} w_{p_{0}}\right) Q_{0_{S}} w_{S_{0}}}
$$

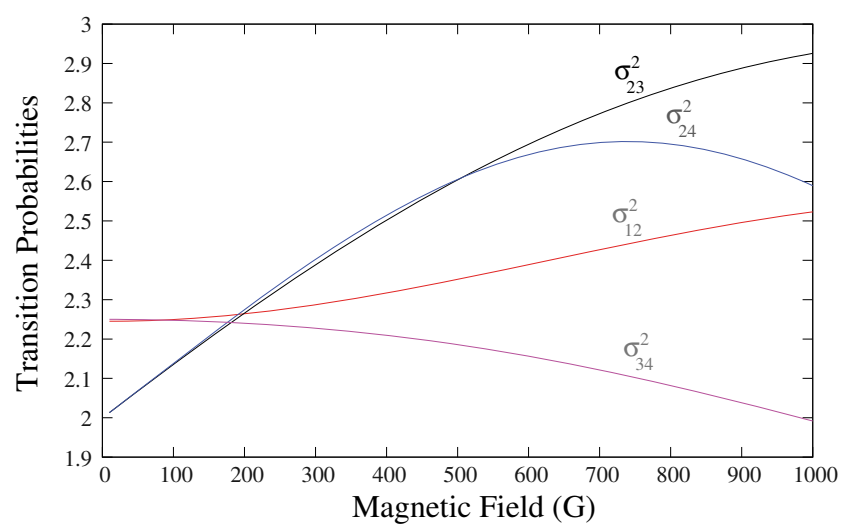

Fig. 5. (Color online) Transition probabilities $\left(\sigma_{i j}^{2}\right)$ between levels $i$ and $j$ as a function of a magnetic field perpendicular to the crystal $Z$-axis.

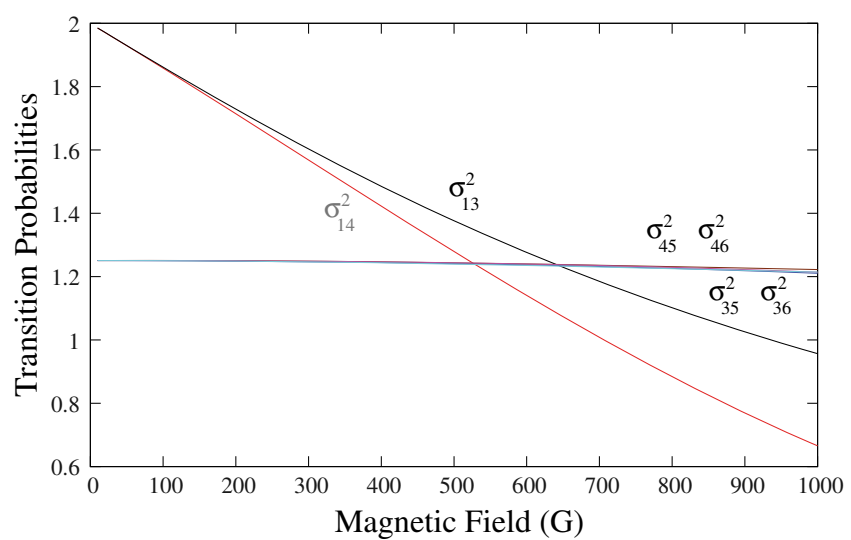

Fig. 6. (Color online) Transition probabilities $\left(\sigma_{i j}^{2}\right)$ between levels $i$ and $j$ as a function of a magnetic field perpendicular to the crystal $Z$-axis.

a Gaussian normal distribution:

$$
g(\nu)=\frac{1}{\sigma \sqrt{2 \pi}} \operatorname{Exp}\left[-\frac{1}{2}\left(\frac{\nu-\nu_{p}}{\sigma}\right)^{2}\right]
$$

with $\sigma^{-1}=2 \tau_{2} \sqrt{2 \pi}$ [10] and where $\tau_{2}$ is the spin-spin relaxation time equal to 1 ns [19]. The line shape function for the pump and the maser signal was calculated, but on the next plot we represent only the line shape function for the pump signal (see Fig. 9).

As the levels 5 and 6 are only separated by $450 \mathrm{kHz}$ the two line shape functions are very similar and confirm that states 5 and 6 are mixed together.

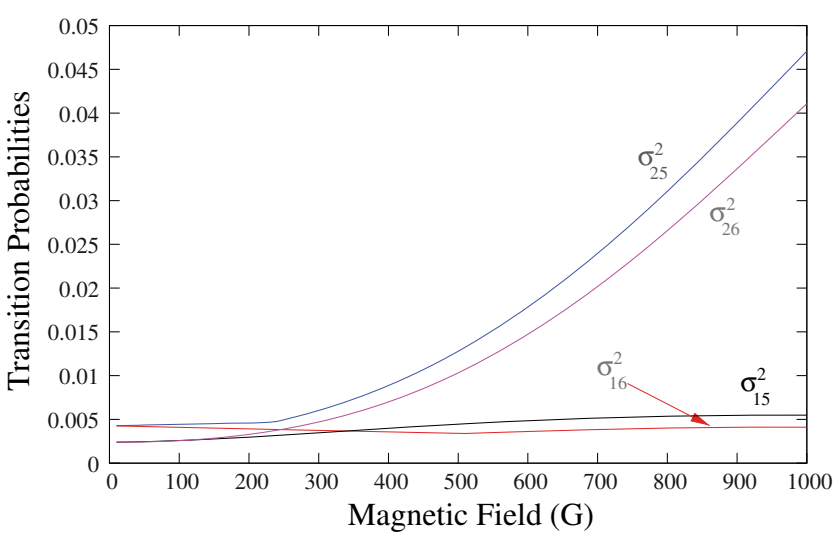

Fig. 7. (Color online) Transition probabilities $\left(\sigma_{i j}^{2}\right)$ between levels $i$ and $j$ as a function of a magnetic field perpendicular to the crystal $Z$-axis.



Fig. 8. (Color online) Transition probabilities between levels 5 and 6 as a function of a magnetic field perpendicular to the crystal Z-axis.

\section{Evolution of the maser output power and pump power threshold}

To determine an order of magnitude of these powers we will consider the parameters at $4.2 \mathrm{~K}$ of one of our resonators [18]: the total population is $N=3.8 \times 10^{20}$ ions $/ \mathrm{m}^{3}$, the effective volumes of the WGM are $V_{14}=$ $V_{p}=11.7 \times 10^{-6} \mathrm{~m}^{3} \cdot Q_{0_{S}}=686 \times 10^{6}, Q_{0_{p}}=10^{8}$ and $Z_{c}=112 \Omega$.

On the two next plots we have represented the evolution of the pump power threshold and the maser output power as a function of the orientation of the magnetic field and its amplitude.

As we can see in Figure 10 the orientation of the magnetic field has no influence on the maser output power 


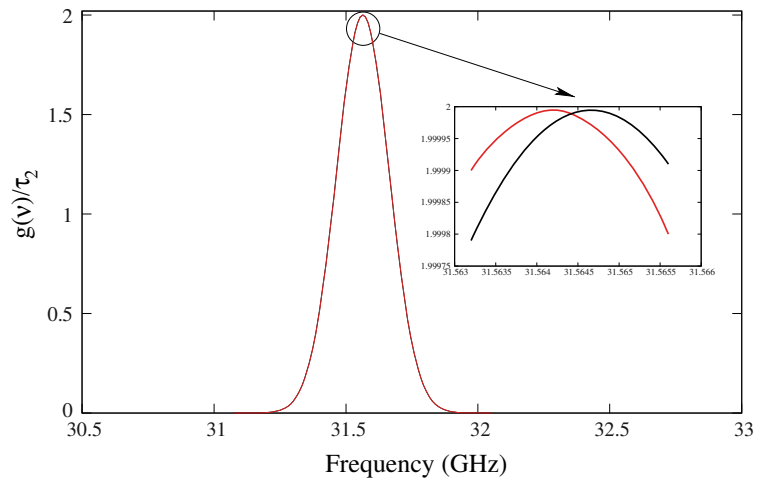

Fig. 9. (Color online) $g\left(\nu_{15}\right)$ and $g\left(\nu_{16}\right)$ as a function of $\nu_{p}$ $(\mathrm{GHz})$ for a magnetic field of $50 \mathrm{G}$ perpendicular to the crystal Z-axis.

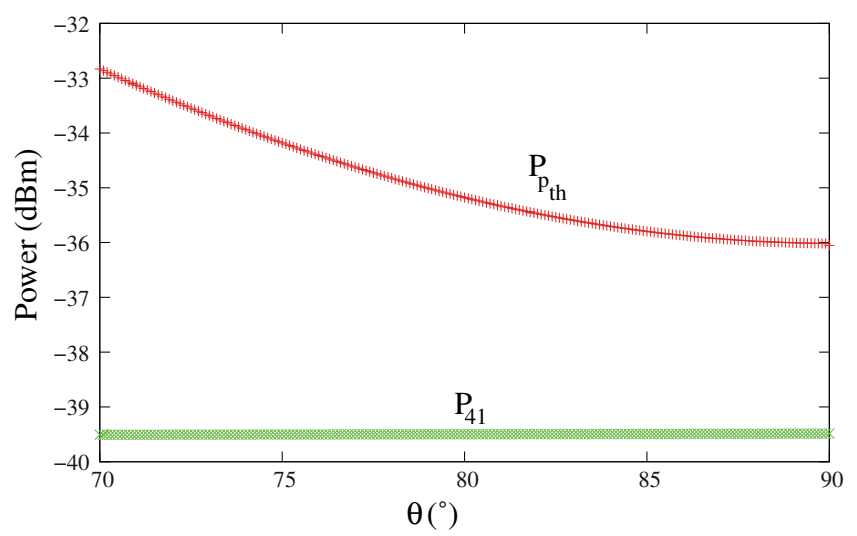

Fig. 10. (Color online) Evolution of the maximum emitted power and pump threshold as a function of the orientation of the magnetic field, for a magnetic field of $50 \mathrm{G}$.

while for the pump power threshold it is minimum for an angle of $90^{\circ}$. Moreover when the magnetic field increases (Fig. 11) the pump power threshold also increases and the maser output power is still constant over $500 \mathrm{G}$ before decreasing. These two curves confirm our choice to work at a low-level magnetic field $(50 \mathrm{G})$ perpendicular to the crystal $Z$-axis.

The last plot represents the comparison between the maser output power without and with a magnetic field $\left(50 \mathrm{G}, 90^{\circ}\right)$ (see Fig. 12).

The use of magnetic field enables us to increase the maser output power of $6 \mathrm{~dB}$.

\section{Conclusion}

A complete model of the Whispering Gallery Mode maser under magnetic field was developed. The eigenvectors and the eigenvalues of the $\mathrm{Fe}^{3+}$ ion Hamiltonian in the sapphire lattice were calculated in order to determine all the transition probabilities, energy levels, etc.

A general relation between the maser output power and the pump power was also determined. From this calculation we have demonstrated that the use of a magnetic

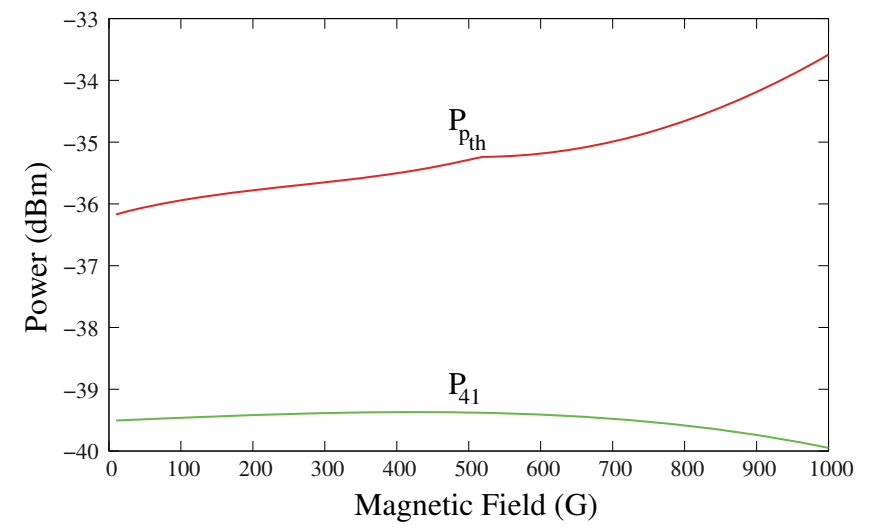

Fig. 11. (Color online) Evolution of the maximum emitted power and pump power threshold as a function of the magnetic field perpendicular to the crystal Z-axis.

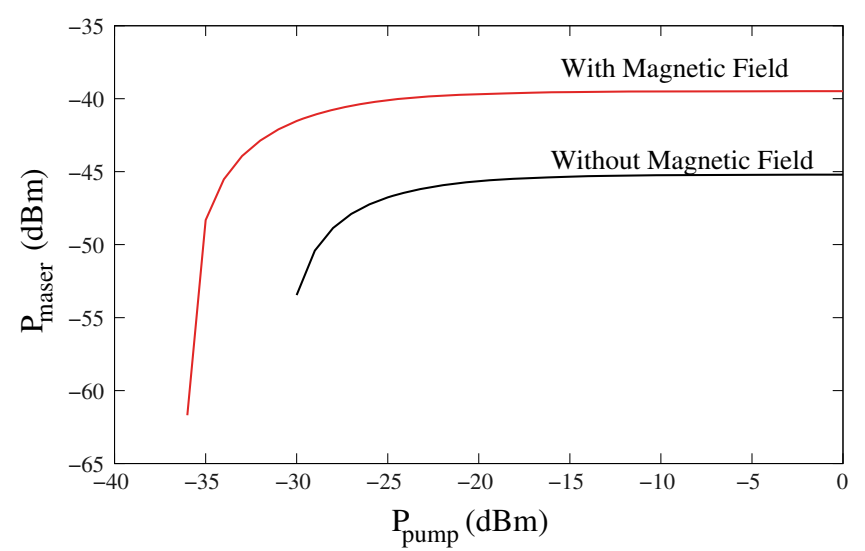

Fig. 12. (Color online) Evolution of the maximum emitted power as a function of the pump power with and without magnetic field. The magnetic field is perpendicular to the crystal $Z$-axis with a value of $50 \mathrm{G}$.

field perpendicular to the crystal axis and with an amplitude of $50 \mathrm{G}$ can increase the maser output power by a factor of $6 \mathrm{~dB}$, and also that the pump power threshold can be reduced.

\section{References}

1. S. Grop, P.-Y. Bourgeois, N. Basin, Y. Kersalé, E. Rubiola, C. Langham, M. Oxborrow, D. Clapton, S. Walker, J. De Vicente, V. Giordano, Rev. Sci. Instrum. 81, 025102 (2010)

2. J.G. Hartnett, C.R. Locke, E.N. Ivanov, M.E. Tobar, P.L. Stanwix, Appl. Phys. Lett. 89, 203513 (2006)

3. P.-Y. Bourgeois, N. Bazin, Y. Kersalé, V. Giordano, M.E. Tobar, M. Oxborrow, Appl. Phys. Lett. 87, 224104 (2005)

4. P.-Y. Bourgeois, M. Oxborrow, M.E. Tobar, N. Bazin, Y. Kersalé, V. Giordano, Int. J. Mod. Phys. B 20, 1606 (2006)

5. K. Benmessai, P.-Y. Bourgeois, Y. Kersalé, N. Bazin, M.E. Tobar, J.G. Hartnett, M. Oxborrow, V. Giordano, Electron. Lett. 43, 1436 (2007) 
6. A.L. Schawlow, C.H. Townes, Phys. Rev. 112, 1940 (1958)

7. N. Bloembergen, Phys. Rev. 104, 324 (1956)

8. G.S. Bogle, H.F. Symmons, Proc. Phys. Soc. 73, 531 (1959)

9. L.S. Kornienko, A.M. Prokhorov, Sov. Phys. JETP 36, 649 (1959)

10. A.E. Siegman, Microwave Solid State Masers (McGraw-Hill, New York, 1964)

11. G.E. Friedman, A.W. Nagy, Proc. IEEE 51, 361 (1963)

12. D.L. Creedon, K. Benmessai, M.E. Tobar, J.G. Hartnett, P.-Y. Bourgeois, Y. Kersalé, J.-M. Le Floch, V. Giordano, IEEE Trans. Ultrason. Ferroelectr. Freq. Control 57, 641 (2010)
13. K. Benmessai, D.L. Creedon, M.E. Tobar, P.-Y. Bourgeois, Y. Kersalé, V. Giordano, Phys. Rev. Lett. 100, 233901 (2008)

14. H.F. Symmons, G.S. Bogle, Proc. Phys. Soc. 79, 468 (1962)

15. H.H. Wickman, G.K. Wertheim, Phys. Rev. 148, 211 (1966)

16. L.S. Kornienko, A.M. Prokhorov, Sov. Phys. JETP 40, 1594 (1961)

17. J.H. Pace, D.F. Sampson, J.S. Thorp, Proc. Phys. Soc. 76, 697 (1961)

18. K. Benmessai, P.-Y. Bourgeois, M.E. Tobar, N. Bazin, Y. Kersalé, V. Giordano, Meas. Sci. Technol. 21, 025902 (2010)

19. P.-Y. Bourgeois, K. Benmessai, M. Oxborrow, M.E. Tobar, N. Bazin, J.G. Hartnett, Y. Kersalé, V. Giordano, in Proc. IEEE IFCS, Genève, CH, 200\%, p. 1031 